\title{
Editorial
}

\section{Quo vadis, cold molecules?}

\author{
J. Doyle ${ }^{1}$, B. Friedrich ${ }^{1,2}$, R.V. Krems ${ }^{3}$, and F. Masnou-Seeuws ${ }^{4}$ \\ 1 Department of Physics, Harvard University, Cambridge, MA 02138, USA \\ 2 Fritz-Haber-Institut der Max-Planck-Gesellschaft, 14195 Berlin, Germany \\ 3 ITAMP, Harvard-Smithsonian Center for Astrophysics, Cambridge, MA 02138, USA \\ 4 Laboratoire Aimé Cotton, CNRS, Bâtiment 505, Campus d'Orsay, 91405 Orsay Cedex, France
}

\begin{abstract}
We give a snapshot of the rapidly developing field of ultracold polar molecules and walk the reader through the papers appearing in this Topical Issue.
\end{abstract}

PACS. 33. Molecular properties and interactions with photons - 33.80.Ps Optical cooling of molecules; trapping - 34. Atomic and molecular collision processes and interactions - 39. Instrumentation and techniques for atomic and molecular physics

\section{Introduction}

The idea to produce this topical issue came to us during a meeting in Cambridge last January, the ITAMP-CUA Workshop on Ultracold Polar Molecules ${ }^{1}$. The ultracold polar weather that beset Cambridge at that time contributed to a congenial and, indeed, warm atmosphere in the halls and hallways of the workshop's venue, the Harvard-Smithsonian Center for Astrophysics.

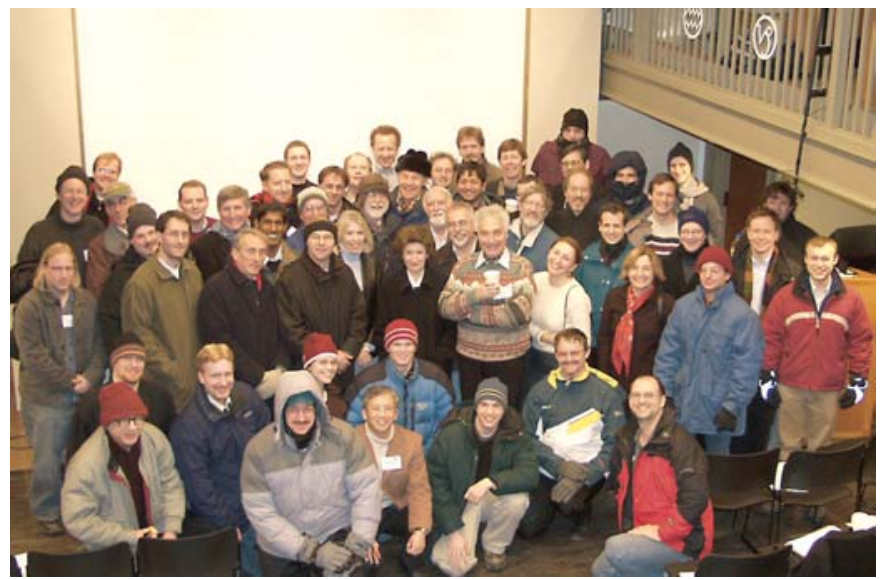

The present collection of papers is a snapshot of a very rapidly developing field. There are now at least nine different techniques used to produce cold molecules, a number that increased from zero over the past eight years.

As cold, we designate molecules with translational temperatures between 1 and $1000 \mathrm{mK}$. Such molecules can be easily manipulated with electromagnetic fields and

1 ITAMP is the Institute for Theoretical, Atomic, Molecular and Optical Physics; CUA is the Harvard/MIT Center for Ultracold Atoms. trapped. As ultracold, we designate molecules whose translational temperatures are typically less than $1 \mathrm{mK}$; such molecules are distinguished by a single partial-wave scattering.

\subsection{Why cold polar molecules?}

Currently, the goal of many researchers is to produce dense samples of ultracold polar molecules. This goal is shared by colleagues with diverse backgrounds, mainly in physical chemistry, chemical physics and in atomic and molecular physics. Why such an intense interdisciplinary interest? Molecules are different from atoms - certainly more complicated - and one might say "more interesting": they offer intriguing properties which are not available with atoms, such as body-fixed electric dipole moments. Hence the emphasis on polar.

As atomic physics has moved beyond the trapping and study of dilute gases of simple atoms, a new manybody physics has emerged. Similarly, fundamental physical chemistry is moving in new directions, becoming increasingly preoccupied with controlling chemical reactions and collisions in general. In both research areas dense samples of cold molecules are called for - and wanting. Once these become available, much is expected to happen: there is the promise of the discovery of new phases of matter, ultracold chemistry, and even a robust quantum computer made out of molecules suspended in free space. These far-reaching research goals and the thrill of the challenge drew a group of about seventy researchers through the ultracold weather to talk about polar molecules. This volume is not a summary of that meeting (please see the Workshop's web page for that, http://itamp.harvard.edu/polar/polar.html) but a series of peer-reviewed articles fostered by that meeting. 
We hope that you will find this collections of papers useful and inspiring.

\subsection{How to create cold polar molecules?}

The discovery of methods for cooling, trapping, and manipulating atoms has generated a revolution in atomic physics. In the past few years many researchers have chosen to pursue the creation and study of ultracold polar molecular gases. Cooling molecules is more difficult than cooling atoms. The complex molecular internal level structure precludes a simple extension of laser cooling to molecules (although work is continuing in this area and the proposals to laser-cool metal hydrides look promising $[1,2])$. Despite hurdles, considerable progress has been made and we are in a period of very rapid world-wide growth in this new field. In 1998, the first trapping of neutral polar molecules, $\mathrm{CaH}$, in a magnetic field was reported [3]. Since then, several groups have made advances in the production of cold polar molecules by a variety of methods. The creation of homonuclear (bosonic) alkali dimers via photoassociation or Feshbach resonances is now being followed up with application of the same techniques to produce polar bi-alkali dimers; recently Feshbach resonances in dual-species traps have been found [4,5]. Ground-state (or at least triplet ground state) bi-alkalis have been produced by photoassociation [6-9]. The methods of photoassociation and Feshbach-resonance linking can be termed "indirect" as the molecules are formed from pre-cooled atoms (see Sect. 2 of this editorial). There are more than twenty groups pursuing the indirect methods and some are turning their attention from homonuclear to polar molecules. The advantages and drawbacks of the indirect methods are described in Section 2.

"Direct" methods are based on cooling preexisting molecules. By current count there are at least fifteen groups pursuing either buffer-gas cooling [3,10-13], electric-field pulsed beam slowing [14-18], light-field slowing [19], laser cooling [1], counter-rotating-nozzle slowing [20], single collision scattering [21], or beam skimming with a guide $[22,23]$. All of these direct methods start with relatively hot molecules, typically from a molecular beam source, and employ some combination of slowing, cooling and trapping as an initial step toward creation of ultracold polar molecular gases (see Sect. 3 of this editorial). The advantages of (many of) the direct approaches include wide applicability and large yield $(\mathrm{CaH}$, $\mathrm{OH}$, and $\mathrm{NH}_{3}$ have been already trapped and a wide variety of molecules, including benzene, have been slowed). A disadvantage is that many of these methods generally do not immediately yield the very low translational temperatures, typically in the microkelvin range, which are attainable with the indirect methods. It will be necessary to bridge the "temperature gap" between the initial loading temperatures of the direct methods $(1-1000 \mathrm{mK})$ and the desirable ultracold regime $<1 \mathrm{mK}$. There are a wide variety of proposals on how to do this, including direct evaporative cooling, sympathetic cooling with laseror evaporatively cooled atoms, or direct laser cooling. The application of these techniques will generally require starting with a large number of molecules. It will also require learning a great deal of new physics.

The payoff should be worth the effort. Most prominently, the large electric dipole moments of polar molecules produce a strong interparticle interaction that can be exploited for quantum computing or as a source for direct BCS pairing [24-29]. Also, precision spectroscopy of heavy dipolar molecules in searches for an elementary-particle electric dipole moment (EDM) $[16,17]$ holds tremendous promise for reaching physics beyond the Standard Model. Producing larger numbers of colder molecules will naturally lead to improvements in these efforts. Beyond these anticipated phenomena, there awaits a terra incognita. What are the possibilities for chemistry at low temperature? Will there be applications to clocks? Are there new collisional phenomena? These are some of the questions that are on the table. Just as we are now seeing phenomena in the ultracold atom field not dreamt about twenty years ago when BEC research was launched, we expect that new and exciting molecular phenomena we can't now imagine will emerge ten to twenty years hence.

\section{Making ultracold polar molecules from cold atoms}

\subsection{Photoassociation and radiative stabilization}

Laser cooling techniques cannot be easily applied to molecules [2,30], which are complex multi-level systems. One can circumvent this difficulty by first cooling the constituent atoms, then making excited state molecules by photoassociation of atom pairs with laser light, following the scheme proposed in 1987 by Thorsheim et al. [31] and discussed in multiple reviews [32-35]. However, molecules thus obtained are short-lived and decay by spontaneous emission, most often dissociating back into atoms. In order to produce stable molecules, population transfer from a bound level $v^{\prime}$ of the excited electronic state to bound levels $v$ of the ground (or lower triplet) electronic state must occur. This stabilization process involves either spontaneous emission, or induced emission with a second laser. The advantage of the photassociation method is that one makes ultracold molecules at the same translational temperatures as the precursor atoms (below $1 \mathrm{mK}$ ). A drawback is that the molecules are often formed in excited vibrational levels of the electronic ground state, so that they are not vibrationally cold. Also, in many cases, the stable molecules are formed in the lower triplet state rather than the ground state and they can be captured due to their low temperature either in a magnetic trap $[33,34]$ or in a dipole trap at the focus of a $\mathrm{CO}_{2}$ laser beam [36].

The first stable ultracold molecules that were formed, detected by photoionization and time-of-flight selection of the molecular ions, were homonuclear alkali dimers [37-43]. Several mechanisms were identified as the stabilization step, all associated with specific properties of the potential curves in the excited electronic state. 
The progress and development of new schemes appear to be strongly dependent on accurate spectroscopic information about the molecules and on vigorous symbiosis between theory and experiment (see the reviews [33,34] and references therein).

As for the formation of heteronuclear dimers, there is an added complication of using a double MOT. This issue is "merely" technical and has been solved by many groups. Regarding the photoassociation itself, it was predicted [44] that the photoassociation step would be less efficient for heteronuclear dimers than for homonuclear molecules. The heteronuclear molecular potentials in the excited electronic state vary asymptotically as $R^{-6}$, while for homonuclear molecules the potentials correlating with the first excited asymptotic limit have a long range $R^{-3}$ behaviour due to the resonant exchange interaction. Since the photoassociation of ultracold atoms occurs at large distances, the $R^{-3}$ potentials, which support bound levels with larger extension, are more favourable. However, the stabilization step is quite efficient for heteronuclear dimers, due to the similar $R^{-6}$ asymptotic behaviour of both the ground and excited potential curves. This makes bound-bound transitions more probable in heteronuclear molecules than in homonuclear dimers. KRb, RbCs and $\mathrm{KCs}$ were predicted to be promising candidates [44]. After successful detection of a signature of stable molecules, molecular ion production $[45,46]$, it took five years to make ultracold heteronuclear dimers with clear identification of the production mechanisms. The following molecules were first formed and state-identified in 2004: RbCs by the Yale group [47,48] and KRb by the UConn ${ }^{2}$ group [6] and by the Sao Paulo group [49]. Contributions from the first two groups are presented in this issue, which contains more than ten papers on the subject of photoassociation.

The UConn group's paper by Wang et al. (pp. 165177) presents a detailed photoassociation spectroscopy of $\mathrm{KRb}$, formation of molecules in the ground triplet state $\mathrm{a}^{3} \Sigma^{+}$, and detection of stable molecules using a twophoton ionization scheme. A wealth of information is thus available on the molecular levels in the range of $95 \mathrm{~cm}^{-1}$ below the $\mathrm{K}(4 s)+\operatorname{Rb}\left(5 p_{1 / 2}\right)$ asymptotic limit, elucidating the interactions and perturbations between various vibrational series. Such a resonant structure was known to be important for the efficiency of the stabilization step (resonant coupling mechanism [42]). The paper shows that it can also be a loss path, through predissociation, since a photoassociated molecule could rapidly dissociate before emitting a photon that would stabilize it. Information on potential curves and transition moments can be obtained from the spectra, yielding useful data to design new formation schemes. The stable KRb molecules can be trapped in a magnetic field, as expected for triplet state molecules, which was already demonstrated for $\mathrm{Cs}_{2}$ [50] and $\mathrm{Rb}_{2}$ [51]

The paper of Bergeman, Kerman, Sage, Sainis, and DeMille (pp. 179-188) investigates the possibility of transferring $\mathrm{RbCs}$ stable molecules from the lower triplet state $\mathrm{a}^{3} \Sigma^{+}$to the ground state $\mathrm{X}^{1} \Sigma^{+}$. Stable molecules are created as in previous experiments $[47,48]$. We should

${ }^{2}$ UConn is the University of Connecticut. note the large value of the densities (close to $10^{12} \mathrm{~cm}^{-3}$ ) for both species, the temperature of $75 \mu \mathrm{K}$, and the very large number of Cs atoms attained in these experiments. The molecules are formed in a bound level of the lower triplet state and they are re-excited by a photon into a bound level of an excited electronic state correlating with the $\mathrm{Rb}(5 s)+\mathrm{Cs}(6 p)$ asymptotic limit. From an analysis of the bound-bound spectrum, data are extracted on the excited potential curves and on the coupling between various channels. Using this information, the best schemes to transfer population from a bound level in an excited electronic state (or in a mixture of such excited states, due to coupling) to a bound level of the ground $\mathrm{X}^{1} \Sigma^{+}$state are discussed. From the Franck-Condon factors, it is shown that populating the $v=0$ and $v=1$ levels is a realistic goal, and that making translationally and rotationally cold molecules that are also in the ground vibronic state should be feasible.

The same problem is addressed theoretically in the paper by Kotochigova, Tiesinga, and Julienne (pp. 189194) for the KRb molecule. The potential curves and transition dipole matrix elements are determined by ab initio calculations. A wide range of transition moments is thus available. The starting point is a pair of $\mathrm{K}(4 s)$ and $\mathrm{Rb}(5 s)$ atoms, spin-polarized and therefore interacting within the $\mathrm{a}^{3} \Sigma^{+}$state. The photoassociation laser creates a resonance with a level $v^{\prime}$ in the excited state $\left(20^{+}\right)$. The latter is such that the transition dipole moment to a vibrational level of the ground $\mathrm{X}^{1} \Sigma^{+}$is also large. This is due to the fine structure coupling in the excited state, where the singlet and triplet states are mixed, so that radiative transitions to both ground and lower triplet states are allowed. The authors note that this is not possible for homonuclear molecules, where the $u-g$ symmetry would forbid one of the transitions. Thus, it is a big advantage of the heteronuclear systems. The authors propose a twocolor Raman photoassociation experiment in which the first photon is slightly detuned from a photoassociation resonance, while the second photon drives the transition from the $\left(20^{+}\right)$level to a vibrational level of the ground state: the numerical calculations show that the latter can be rather low $(v<30)$ and propose optimal paths. Using the computed values for the permanent dipole moments in the ground state, the authors discuss the stability of heteronuclear molecules relative to thermal blackbody radiation, which would cause cascading from one vibrational level to another in the same electronic state. However, the lifetimes are found to be much longer than the typical duration of an experiment, in agreement with earlier calculations [52].

Is it possible to go beyond the favorite molecules RbCs and KRb? The theoretical paper by Azizi, Aymar, and Dulieu (pp. 195-203) assesses photoassociation and cold molecule formation rates, which are computed for all the heteronuclear dimers composed of either $\mathrm{Rb}$ or Cs atoms. As in the previous paper, the rates are computed from numerical vibrational wavefunctions and the integral over the dipole transition moment rather than estimates of the probability for vertical transitions. The results are 
compared to the homonuclear $\mathrm{Cs}_{2}$ rates, using a rescaling factor for the differences in reduced mass and atomic Rabi frequency. It is shown that the photoassociation rates are reduced by a factor $\approx 30$ for a pair of identical atoms compared to a pair of different atoms. However, since for heteronuclear dimer molecules the excited potential curves have the same $R^{-6}$ asymptotic behavior as the ground or lower triplet curves, the radiative decay to bound levels is faster than in homonuclear molecules, making the stable molecule formation rates only one order of magnitude smaller than for $\mathrm{Cs}_{2}$. Another conclusion is that the lighter species (LiRb, NaRb, LiCs, NaCs) should be much better candidates than previously thought, therefore encouraging experiments in that direction.

As for $\mathrm{NaCs}$, detailed information, necessary to design new schemes, is now available from the extensive spectroscopic work of Docenko, Tamanis, Ferber, Pashov, Knöckel, and Tiemann (pp. 205-211), from Riga and Hannover. In the paper published here, the molecular spectroscopy experiment is described and a very accurate determination of the $\mathrm{X}^{1} \Sigma^{+}$potential curve is presented, by fitting an analytical function to the measured energies of more than 2000 rovibrational levels. The long range coefficients such as $C_{6}$ can thus be determined very precisely. The spectrum provides information on the shallow $\mathrm{a}^{3} \Sigma^{+}$ potential and the authors estimate the scattering lengths for both potentials.

Another candidate appears to be the $\mathrm{LiH}$ molecule, as discussed in the paper of Taylor-Juarros, Côté, and Kirby (pp. 213-219). The dipole moment of the alkali hydride molecules can be larger than that of heteronuclear alkali dimers. As in the two theoretical papers described above, the authors compute the photoassociation rates from a continuum level to a bound level $v^{\prime}$ of the $\mathrm{A}^{1} \Sigma^{+}$ excited state, followed by stimulated emission rates to a bound level $v$ of the ground $\mathrm{X}^{1} \Sigma^{+}$state, looking for the best $v, v^{\prime}$ combination for a stimulated Raman photoassociation experiment. Important formation rates are found for stable molecules. An advantage of the alkali hydrides is that due to the large dipole moment in the $\mathrm{X}^{1} \Sigma^{+}$state (typically one order of magnitude larger than in the KRb molecule discussed above) the stable molecules formed in an excited $v$ level decay by spontaneous emission to the ground vibrational level $v=0$ with relatively short lifetimes.

The Raman scheme is also discussed by Stwalley (pp. 221-225) with the aim of converting molecules formed by Feshbach resonances into ground-state, $v=0, J=0$ molecules. The initial state is considered to be a loosely bound level of ${ }^{3} \Sigma^{+}$symmetry rather than the continuum, corresponding to recent experiments in condensates with magnetically tuned Feshbach resonances. It could also be populated by photoassociation followed by spontaneous emission. Besides KRb, the molecules discussed from a general perspective are $\mathrm{LiNa}, \mathrm{NaK}, \mathrm{NaRb}$, and RbCs. The region of the spectrum where detailed studies should be performed is indicated.

Up to now, photoassociation has been used to populate bound levels in the electronic states correlating with the first excited atomic limit. It was often suggested that highly excited molecules at intermediate and long range were also good candidates [53-55]. Alternatively, the initial state can also be a continuum state of an excited potential curve: a very interesting experiment was recently performed by the $\mathrm{ENS}^{3}$ group in Paris [56]. Starting from spin-polarized metastable helium $\left({ }^{4} \mathrm{He}\right)$ atoms at very low temperatures ( 2 to $30 \mu \mathrm{K}$ ), bound levels of electronic states correlating with the $2^{3} \mathrm{~S}_{1}+2^{3} \mathrm{P}_{0}$ asymptotic limit are populated by photoassociation. Details are presented here by Kim, Rapol, Moal, Léonard, Walhout, and Leduc (pp. 227-237). Such molecules should decay by Penning ionization, but for some symmetries $\left(0_{u}^{+}, 1_{u}\right)$ the existence of long range wells constrains the relative motion to very large interatomic separation, with the inner turning point around $150 a_{0}$ (while the outer turning point can be as far as $\left.1100 a_{0}\right)$. Penning ionization is therefore inhibited, as photoionization was inhibited in reference [53]. The novelty of the experiment is in the calorimetric detection of the photoassociation resonance: when the laser frequency is tuned to a molecular transition, creating a bound photoassociated molecule, the latter decays by spontaneous emission, yielding a pair of atoms that heat the cloud of metastable atoms. This increase of temperature can be detected by optical methods. Very accurate photoassociation spectra are thus produced, with line widths of a few $\mathrm{MHz}$. Excellent agreement is obtained between the observed spectrum and calculations based on long-range potentials. Future work will explore the magnitude of the scattering length by accurate photoassociation spectroscopy.

All the papers described so far have treated the laser coupling in the photoassociation reaction in a perturbative way. In fact, laser light can also be used to manipulate molecules, control the photoassociation reaction or tune the scattering length. The concept of an optically induced Feshbach resonance is very useful for understanding such physical processes. We will discuss optically induced Feshbach resonances in this section and magnetically induced Feshbach resonances in Section 2.1.

The theoretical paper of Luc-Koenig, Vatasescu, and Masnou-Seeuws (pp. 239-262) discusses the possibility of replacing cw lasers by a series of chirped laser pulses which optimize both the photoassociation rate and the formation of stable molecules in low vibrational levels. A time-dependent non-perturbative treatment of the photoassociation reaction in a cold cesium sample has been proposed earlier in 2004 [57]. In the present paper the authors discuss the choice of the parameters of the chirped pulses (intensity, duration, linear chirp rate). The variation of the laser frequency allows one to sweep through several optical Feshbach resonances, determining a photoassociation window within which complete adiabatic population transfer from the ground electronic state to bound levels of the excited state is taking place. A large number of vibrational levels can be populated coherently, so that with a reasonable repetition rate of the laser pulses, the number of molecules formed per unit

\footnotetext{
${ }^{3}$ ENS is École Normale Supérieure.
} 
time is expected to be much larger than with a cw laser of equivalent power. Besides, revival phenomena can occur for the vibrational motion of long-range molecules. It is therefore possible to compute the linear chirp parameter in order to create, within the excited state, a wavepacket focused at the inner turning point of a long range well. This wavepacket has a large Franck-Condon overlap with low vibrational levels of the ground state, so that a second laser pulse could create vibrationally cold stable molecules. The method is similar to the Raman scheme discussed in many papers of this issue but uses pulsed lasers. The avantage of short pulses is that stable molecules in high vibrational levels can be transferred to $v=0$ in very short times, shorter than collisional relaxation or characteristic dissociation times [58].

Manipulating the scattering length with a photoassociation laser has been proposed in several theoretical papers [59-61], and achieved in the experiments of references [62,63]. The paper by Koelemeij and Leduc (pp. 263-271) analyzes the possibility of using photoassociation to determine the scattering length of metastable helium (through accurate measurement of the binding energy of the last bound level of the ground state) and to manipulate it in order to control the dynamics of metastable helium condensates.

A generalized theory of photoassociation in quantum degenerate gases is presented in this issue by Mackie, Dannenberg, Piilo, Suominen, and Javanainen (pp. 273-282). In particular, the authors discuss photoassociation (together with magnetic Feshbach resonances, see below Sect. 2.2) in mixed Fermi-Bose gases. They report a numerical observation of large-amplitude atommolecule oscillations similar to those occurring in pure Bose gases. The authors also discuss the prospects for fermion atom-fermion molecule pairing and explore the transition from triatomic molecules to correlated atommolecule pairs.

We have already mentioned that photoassociation leads to long-range molecules. The vibrational motion of long-range molecules is determined by long-range interatomic forces which can be effectively described by theoretical models such as generalized quantum defect theory. The cold molecule research field is therefore linked to the field of Rydberg atoms, and many concepts are similar (we have mentioned earlier scaling laws and revival phenomena) with differences arising from the $R^{-n}, n=3,6$, rather than $R^{-1}$ asymptotic behaviour. This very fertile topic is represented here by the paper of Gao (pp. 283289 ), which reports systematics of rotational energy levels of diatomic molecules in the vicinity of a dissociation threshold. A new classification scheme of long-range states of diatomic molecules is proposed. The study should be helpful for the analysis of photoassociation spectra and for the determination of scattering lengths from the spectroscopy of the last vibrational levels (see the two papers on metastable helium quoted above, Ref. [32] and references therein).

Another aspect of the theoretical developments in the cold-molecule field, where the relative motion of two atoms has to be accurately described from a distance of around one to several thousands of atomic units, is the implementation of numerical grid methods to represent vibrational or continuum wavefunctions, with grid steps adapted to the local de Broglie wavelength: such methods are routinely used in at least six papers in this volume.

\subsection{Making cold molecules from cold atoms via magnetic Feshbach Resonances}

The possibility of creating ultracold molecular BoseEinstein condensates via resonant collisions [64-76] became a reality in 2003 [77-80]. The idea is simple but its realization is not, as a very dense gas of quantumdegenerate (or near-degenerate) precursor atoms is required. Fortunately, technology has advanced enough so that ultracold alkali metal atom gases are now routinely produced by more than 30 groups worldwide.

In order to stitch ultracold atoms together and create molecules, a Feshbach resonance can be used. The reader is directed to other publications ([29] and references therein) for a more detailed picture. In brief, in the ultracold regime the collision of two atoms is described by the scattering length. This scattering length is very sensitive to the interaction (molecular) potential. If the atoms or the molecule or both are paramagnetic, the potential can be tuned by an external magnetic field. A Feshbach resonance occurs when the energy of the unbound state (the atomic pair) and of the bound state (the diatomic molecule) become equal. The scattering length changes at a Feshbach resonance. It can be made positive or negative and its magnitude arbitrarily large (within the limits of unitarity). By tuning through the resonance the atom pair can be driven into the bound molecular state. The binding energy of the molecule is tiny, typically in the $\mathrm{mK}$ range. Molecules created from bosonic atoms are quickly destroyed by collisions, while molecules produced from fermionic atoms may exist for a long time [68-70]. It is believed that it might be possible to transfer them to more tightly bound states using, for example, pulsed Raman laser fields [58] (see also the discussion in Sect. 2.1). At the workshop, this approach to producing heteronuclear molecules was discussed but no experimental results were then available. Now there are at least two groups that have found Feshbach resonances in heteronuclear systems $[4,5]$. This greatly raised the expectation that ultracold polar molecules will be produced in this way.

The rich resonant structure of molecular spectra observed in ultracold collision experiments cannot be explained without a rigorous theoretical analysis. Bhattacharya, Baksmaty, Weiss, and Bigelow (pp. 301-306) present an article in this issue with a clear discussion of methods for calculating Feshbach resonances in heteronuclear alkali dimers. The presentation is both simple enough to be understood by a general reader and comprehensive enough to be of interest to an experimentalist. 


\section{Methods for cooling, slowing, and trapping polar molecules}

(a) Buffer-gas cooling is a versatile technique which provides the means to cool any molecules (or atoms) to temperatures of a few hundred millikelvin. This temperature is determined by the equilibrium vapor pressure of the He buffer gas that is used to thermalize the molecules via elastic collisions. The technique, pioneered by the Doyle group in 1997 [81], has been used in a number of studies since to cool paramagnetic atoms or molecules and to load them into a magnetic trap [82]. A recalcitrant problem has been the introduction of the molecules to be cooled into the cryogenic buffer gas. Solving this problem has been of primary importance, since the number of molecules that can be cooled and trapped is limited mainly by the number of molecules that can be brought into the buffer gas [3]. The techniques that have been applied so far, laser ablation [82] and capillary filling [83], suffer from serious drawbacks. Capillary filling is suitable only for stable molecules that remain gaseous at low temperatures. Laser ablation offers more versatility (including in situ production of unstable species), but the yield and variety of molecules produced from a solid precursor and, indeed, the precursor's very chemical identity are hard to predict.

The paper by Egorov, Campbell, Friedrich, Maxwell, Tsikata, van Buuren, and Doyle (pp. 307311) describes a new technique of injecting molecules into the buffer gas, one which offers significant advantages over the existing techniques. The reported technique makes use of a molecular beam made up of the species of interest and employs the beam to deliver this species into the cryogenic cell. The work demonstrates that a subtle balance can be struck between the molecular beam flux into the cell and the flux of the He buffer gas out of the cell such that the injection and thermalization occur on time scales shorter than the diffusion loss time. The beam-loaded buffer gas cooling technique is demonstrated with a pulsed beam of $\mathrm{NH}$ radicals produced from $\mathrm{NH}_{3}$ in a glow discharge. The maximum number of ground-state (i.e., thermalized) $\mathrm{NH}\left({ }^{3} \Sigma^{-}, v=0, N=0\right)$ molecules loaded into the cell of volume of $150 \mathrm{~cm}^{3}$ was measured to be $10^{12}$. The radicals were detected via laser-induced fluorescence. In addition, the authors make the point that the molecular beam can be rid of unwanted species by applying standard electrostatic or magnetic filters.

(b) Stark acceleration or deceleration provides the means to alter the velocity of polar molecules at will and to simultaneously select the molecules' internal states. Although conceived almost half a century ago, the technique was implemented only recently, in 1999, by the group of Meijer [84]. The technique relies on timedependent inhomogeneous fields generated by an array of field stages, to repetitively alter the Stark potential energy of the molecules and, by energy conservation, also their kinetic energy. In a Stark decelerator, the potential energy is increased at each decelerator stage and thus the kinetic energy is correspondingly reduced (and vice versa in an accelerator). In order to accelerate/decelerate a bunch of molecules with a finite distribution of positions and velocities, the acceleration/deceleration process must take place under the condition of phase stability, much appreciated in charged-particle accelerator physics $[85,86]$. Stark deceleration was first demonstrated with an array of 63 pulsed electric field stages. This was shown to decelerate metastable CO molecules from $225 \mathrm{~m} / \mathrm{s}$ to $98 \mathrm{~m} / \mathrm{s}$ [84].

Since 1999, the Stark decelerator underwent many refinements and was combined with other devices [87], most notably a trap [88], a buncher, and a storage ring [89] (see below). Also other laboratories have since implemented Stark deceleration [17,90-94].

Friedrich's paper (pp. 313-336) provides a rigorous description of the dynamics of molecules in a Stark accelerator/decelerator. The paper opens with a Fourier analysis of the time-dependent inhomogeneous Stark field, of the kind generated in current experiments by linear switchable field arrays [95]. This is used as a basis for developing a quasi-analytic model of a Stark accelerator/decelerator. This model depends on just a small number of parameters; the lowest-order version (referred to as the firstharmonic accelerator/decelerator model) depends on a single Fourier coefficient, one which accounts for the spatial dependence of the inhomogeneous electric field. Yet, the simplest version of the model is shown to explain the key features of the accelerator/decelerator (such as phase stability and longitudinal acceptance), and to capture the dynamics of the acceleration/deceleration process semiquantitatively. An extensive discussion of the dynamics exploits the isomorphism of the first-harmonic accelerator/decelerator with a biased pendulum. Also, the general properties of the velocity of molecules in a phase-stable accelerator/decelerator are examined, and found to reveal a similarity between a Stark accelerator/decelerator and a flying accordion.

The paper by van Veldhoven, Küpper, Bethlem, Sartakov, van Roij, and Meijer (pp. 337-349), demonstrates the benefits of using slow molecules in microwaveUV double-resonance spectroscopy. The resolution of the inversion tunneling spectrum of ${ }^{15} \mathrm{ND}_{3}$ is shown to be boosted by Stark-decelerating the molecules to a velocity of $52 \mathrm{~m} / \mathrm{s}$. The transit-time broadening is thereby reduced by a factor of 10 compared with undecelerated molecules moving at $280 \mathrm{~m} / \mathrm{s}$. This results in a fully resolved hyperfine spectrum with a $1 \mathrm{kHz}$ linewidth (the spectrum was taken for the $J=1, K=1$ state over $1.43 \mathrm{GHz}$ ). A simulation of the measured spectrum, based on a generalized tensor coupling scheme, is also described. As the authors point out, the inversion frequency in ammonia depends sensitively on the electron/proton mass ratio, and could be used to monitor the ratio's possible time dependence. In order for the techniques to become competitive [96], the present accuracy needs to be further improved, by a factor of $10^{6}$. This should become possible once an ammonia molecular fountain is implemented, allowing for an interaction time of as much as a second. We note that deuterated ammonia molecules, due to their small inversion splitting, exhibit a first-order Stark effect at "all" 
field strengths (including the low ones). This makes them a favorite choice for studies on molecular manipulation.

The paper by Hudson, Bochinski, Lewandowski, Sawyer, and Ye (pp. 351-358) analyzes the experimental conditions needed for an efficient Stark deceleration of a beam of $\mathrm{OH}$ radicals. Calculations are presented that show the fraction of molecules decelerated, given a certain emittance of the molecular beam and acceptance of the decelerator. The calculated fraction is compared with experimental data, and a good agreement is found. The fraction decreases with the decreasing goal velocity and drops to about $0.1 \%$ for a goal velocity of about $30 \mathrm{~m} / \mathrm{s}$, for molecules moving initially at $370 \mathrm{~m} / \mathrm{s}$, and originating from a hot beam (whose mean velocity and velocity width are, respectively, $415 \mathrm{~m} / \mathrm{s}$ and $90 \mathrm{~m} / \mathrm{s}$ ). The paper makes the point that the fraction of molecules decelerated could be substantially increased if a cold source were used.

(c) A storage ring offers an alternative to trapping. Unlike a trap, a storage ring can accommodate molecules whose longitudinal velocities are not all that low. However, since the ring radius scales with the square of the velocity, table-top storage rings require samples of molecules slowed down to velocities on the order of $10 \mathrm{~m} / \mathrm{s}$. A prototype storage ring for low-field seeking states of polar molecules was implemented by Crompvoets et al. [89] and used to store about $10^{6} \mathrm{ND}_{3}$ molecules for up to $430 \mathrm{~ms}$ corresponding to 50 round trips (the storage time was limited by background gas scattering). The storage ring of a $25 \mathrm{~cm}$ diameter was made up of a hexapole lens bent onto itself. Before loading, the molecules were Stark-decelerated to a velocity of $92 \mathrm{~m} / \mathrm{s}$. In addition, a buncher was implemented, to narrow the longitudinal velocity spread of the decelerated molecules to about $300 \mu \mathrm{K}$.

Nishimura, Lambertson, Kalnins, and Gould (pp. 359-364) propose a storage ring for high-field seekers. The authors envision a storage ring $3 \mathrm{~m}$ in diameter, consisting of eight octants. Each octant contains a buncher and a pair of alternating-gradient focusing triplet lenses. In order to obtain net focusing in both transverse directions, the lenses are arranged in a sequence with gradients alternating in sign (the so called alternating-gradient focusing [97]). Simulations indicate that such a ring would store, e.g., ground-state $\mathrm{CH}_{3} \mathrm{~F}$ molecules with a longitudinal kinetic energy of $2 \mathrm{~K}$ for several minutes. The molecules would be produced in a pulsed supersonic expansion and Stark decelerated before injection into the ring. For a peak beam intensity of $10^{19} \mathrm{sr}^{-1} \mathrm{~s}^{-1}$ and a nearly perfect phase-space matching between the beam, decelerator and ring, each bunch in the ring would consist of about $10^{8}$ molecules and would have a density on the order of $10^{9} \mathrm{~cm}^{-3}$ in the straight sections throughout the storage time. Such a high density may foster evaporative cooling. Apart from storing singular bunches, the ring may be able to hold up to 200 bunches simultaneously. This would provide a large flux desirable, for instance, for collisional or spectroscopic experiments. These could be carried out without field perturbations, in the field-free regions of the storage ring. (d) The paper by Junglen, Rieger, Rangwala, Pinkse, and Rempe (pp. 365-373) describes an electrostatic guide for slow polar molecules in low-field seeking states, which shares some of its key features with the storage ring of Crompvoets et al. [89]. A guide for molecules may find use in molecular interferometry and in the study of slow collisions.

The technique selects the low-velocity tail of a Maxwell-Boltzmann velocity distribution present in a thermal effusive molecular beam. Transverse velocity selection is achieved by injecting the molecular beam into a quadrupole electrostatic field generated by rod electrodes aligned along the beam axis. By bending the rod electrodes, also longitudinal velocity filtering is achieved. The technique was demonstrated with $\mathrm{ND}_{3}$ molecules. A flux of $10^{10}$ translationally cold $\mathrm{ND}_{3}$ molecules per second could be achieved. The longitudinal temperature of the molecules was measured, via time-of-flight mass spectrometry, to be about $4 \mathrm{~K}$. The efficiency of the technique benefits from cooling the beam source. However, the effusive flow regime needs to be maintained, in order for the hydrodynamic velocity to remain negligible and the lowvelocity tail in place. This of course precludes cooling of the internal degrees of freedom of the molecules. Although the Stark effect is somewhat greater for low rotational states, these are not selected efficiently. As a result, the internal temperature of the translationally cold molecules picked up by the Stark field remains close to that of the source.

(e) The Earnshaw theorem precludes trapping of atoms or molecules via their high-field seeking states in static electric or magnetic fields [97]. Since ground states of atoms and molecules are always high-field seeking, there has been a considerable effort to find ways of circumventing Earnshaw's curse, by devising traps for high-field seekers made up of time-dependent electromagnetic fields. These comprise switched static fields [98] (ac traps) or radiative fields, both resonant (MOT's) and nonresonant (optical dipole traps) [78,99]. A common drawback of these traps is their small volume and depth; these significantly limit the ability to apply evaporative cooling which could otherwise be used to drive the trapped molecules into the ultracold regime $(\leq 1 \mathrm{mK})$.

DeMille, Glenn, and Petricka (pp. 375-384) propose a deep, large-volume microwave trap for high-field seeking states of cold/slow polar molecules. A maximum of free-space electric field strength is produced in a confocal cavity, pumped by $2 \mathrm{~kW}$ of resonantly coupled microwave power. Field strengths on the order of $10 \mathrm{kV} / \mathrm{cm}$ in the $\mathrm{TEM}_{00}$ mode spread over a volume of $1 \mathrm{~cm}^{3}$ seem feasible. The trapping is based on a near-resonant electric-dipole interaction of the microwave field with the rotational ground state of a polar molecule. The interaction couples the ground state to higher rotational states and modifies its eigenenergy. For a red detuning of the microwave field, the interaction is attractive and so the eigenenergy drops with respect to the field-free value. For a linear polarization of the microwave field, multiphoton transitions complicate the resulting energy-level pattern. However, 
for a circular polarization (and red detuning), multiphoton transitions can no longer occur, and the energy level pattern greatly simplifies. In particular, the ground-state level clears of avoided crossings with other levels and its dependence on the strength of the microwave field becomes similar to that of a ground state polar molecule in a DC field. Thus, the ground-state eigenenergy of a polar molecule in a red-detuned circularly polarized microwave field can be used for stable trapping. Because of the long radiative lifetimes of the excited rotational states, the red detuning can be minimized, without the danger of heating the trapped molecules by photon scattering (which bedevils trapping at optical frequencies). This maximizes the drop of the eigenenergy with field strength and thus the trap depth. Indeed, according to the dressed-state analysis presented in the paper, the trap depth for the molecular ground state scales roughly as $\mu \varepsilon_{0} / 2$, where $\mu$ is the body-fixed electric dipole moment of the molecule and $\varepsilon_{0}$ the effective field strength at the center of the trap. Hence for $\mu \approx 1$ Debye and $\varepsilon_{0} \approx 10 \mathrm{kV} / \mathrm{cm}$, the expected trap depth is on the order of $100 \mathrm{mK}$.

The paper by DeMille et al. also discusses various options of accumulating and cooling the molecules in the microwave trap. Among these, the possibility of loading multiple pulses of molecules in a low-field seeking state and pumping them into the trappable ground state seems particularly appealing (a similar scheme has been proposed earlier by van der Meerakker et al. [100]). Another cooling scheme envisioned is evaporative cooling driven by elastic collisions. The elastic collision cross-sections are shown to be dramatically enhanced by the alignment of the molecular dipoles by the trapping field and the ensuing strong dipolar interaction. Elastic cross-sections for collisions between ground state $\mathrm{SrO}$ molecules are estimated to come close to $10^{7} \AA^{2}$. This would suffice for a thermalization of the trapped molecules at number densities as low as $10^{8} \mathrm{~cm}^{-3}$.

(f) Single-collision slowing in crossed molecular beams. The paper by Elioff, Valentini, and Chandler (pp. 385393) describes an experiment that demonstrates a rather general technique of producing slow molecules, by billiardlike collisions in crossed molecular beams. Significant numbers of slow NO molecules $(\approx 15 \mathrm{~m} / \mathrm{s}$, corresponding to a translational temperature of about $0.5 \mathrm{~K}$ ) in a single internal state $\left({ }^{2} \Pi_{1 / 2}, v=0, J=15 / 2\right)$ are generated by scattering of a pulsed molecular beam of NO by a beam of Ar. The number of slow molecules produced $\left(\approx 10^{5}\right.$ per pulse) corresponds to a fraction of about $10^{-5}$ of the overall number of molecules that are colliding during a beam pulse. The technique relies on the cancellation of the laboratory velocity of the scattered molecules. This occurs when the laboratory velocity of the center of mass of the colliding partners $(\mathrm{NO}+\mathrm{Ar})$ becomes equal in magnitude but opposite in direction to the center-of-mass recoil velocity of the scattered molecule (NO). The velocity spread of the molecules that satisfy this cancellation condition depends on the velocity spread of the NO beam. For the scattering of partners with the same mass, the condition is fulfilled for elastic collisions, in which case, interestingly, the energy spread of the slow molecules is found to decrease to zero. The technique has been demonstrated using a state-of-the-art detection scheme based on state-specific REMPI ionization and micro-channel plate imaging of the $\mathrm{NO}^{+}$ions produced.

(g) A class of atoms, best represented by the alkalis, can be efficiently cooled by repetitive absorption of photons that counter-propagate with respect to the atoms (Doppler cooling). Since, in the visible range, the momentum transferred by a single photon is small, typically thousands of such photons need to be absorbed before a thermal alkali atom can be brought to a halt. In order for the absorption to be repetitive, the re-emission of the absorbed photon must send the atom into the same initial state where it started. Hence we speak of a closed absorption/emission cycle. Finding molecules with a closed absorption/emission cycle is difficult, if not impossible. As a way out, Bahns et al. have proposed a sequential scheme which uses an array of stimulated Raman sidebands to repump molecules that have fallen out from the cooling cycle [2].

Di Rosa's paper (pp. 395-402) examines whether a certain class of molecules can be laser-cooled without extensive repumping and, provided the molecules are paramagnetic, whether they can be trapped in a magnetooptical trap (MOT). The paper identifies the criteria that molecules must meet in order to be amenable to Doppler cooling and provides a list of such molecules. The criteria include a strong vibronic transition, with a near-unit Franck-Condon factor, and no intervening electronic transitions that could divert photons from a closed cooling absorption/emission cycle. Out of a list of ten diatomic molecular candidates (six of which are paramagnetic), Di Rosa chose $\mathrm{CaH}\left(\mathrm{A}^{2} \Pi_{1 / 2}-\mathrm{X}^{2} \Sigma^{+}\right)$as a representative example.

The number of absorption/emission cycles needed to decelerate a molecule of mass $m$ from an initial velocity $v$ to a standstill with radiation of wavelength $\lambda$ is $N=$ $m v \lambda / h$, which comes close to 4500 for $\mathrm{CaH}\left(\mathrm{A}, v^{\prime}=0\right.$ $\left.\mathrm{X}, v^{\prime \prime}=0\right)$ moving initially at $v \approx 100 \mathrm{~m} / \mathrm{s}$. For a cycling transition with a Franck-Condon factor of 0.9995 , the probability, $p$, of spontaneous emission to levels outside of the absorption/emission cycle is then $p=0.0005$. Hence the probability that a molecule will remain in the absorption/emission cycle is $(1-p)^{N} \approx 0.1$ and so about $10 \%$ of the molecules will be cooled. Although a FranckCondon factor of about 0.9995 is quite rare [100], in some molecules, such as $\mathrm{CaH}(\mathrm{A}-\mathrm{X})$, the Franck-Condon factors for two neighboring levels, $v^{\prime}=0,1 \rightarrow v^{\prime \prime}=0$, add up to a value close to 0.9995 . This would then require a single repumping laser, to drive the $v^{\prime}=1 \rightarrow v^{\prime \prime}=0$ transition.

The experimental work reported by Di Rosa is a first step towards a $\mathrm{CaH}$ MOT. It examines experimentally a laser-cooling cycle for $\mathrm{CaH}$, in particular the underlying hyperfine structure of the $\mathrm{A}-\mathrm{X}$ band. He estimates that the lifetime in a $\mathrm{CaH}$ MOT will be about $1 \mathrm{~ms}$. At a thermal loading rate of the $\mathrm{CaH}$ vapor, the MOT could hold about $10^{5}$ molecules. 
(h) The work of Bertelsen, Vogelius, Jorgensen, Kosloff, and Drewsen (pp. 403-408) takes advantage of the low temperatures $(\approx 10 \mathrm{mK})$ available in Coulomb crystals to control the photodissociation dynamics of $\mathrm{MgH}^{+}$ions embedded in the crystal. The molecular ions are sympathetically cooled into the crystal by laser-cooled atomic ions. The authors foresee that the cold crystal environment along with the tight spatial localization $(\approx 1 \mu \mathrm{m})$ will make it possible to control the branching ratio between the $\mathrm{Mg}^{+}(3 p)+\mathrm{H}$ and $\mathrm{Mg}\left(3 s^{2}\right)+\mathrm{H}^{+}$dissociation channels, by radiative coupling of $\mathrm{MgH}^{+}$(A) to its $\mathrm{B}$ and $\mathrm{C}$ electronic states. This will be effected by two independent laser fields. The paper presents calculations which indicate that the branching ratio could be varied at will, either by varying the intensity of the lasers involved or the frequency of one of the lasers.

(i) Another technique for producing cold molecules relies on thermalization in the cryogenic environment provided by helium nanodroplets. This technique, pioneered in 1992 [101], has been implemented by several groups and used to improve the resolution of molecular spectroscopy $[102,103]$, study nanoscopic superfluidity $[104,105]$ and to foster self-assembly of non-equilibrium structures [106], to name just a few.

He nanodroplets are produced by a supersonic expansion of He at high pressures (5-45 bar) and low temperatures $(5-25 \mathrm{~K})$. The nanodroplets cool down by evaporation to a temperature of $380 \mathrm{mK}$ or $150 \mathrm{mK}$ if ${ }^{4} \mathrm{He}$ or ${ }^{3} \mathrm{He}$ are used, respectively; the uniform temperature of the nanodroplets is determined essentially by their surface tension. The resulting size distribution is log-normal, peaking typically at thousands of He atoms.

The nanodroplets can be doped by atoms or molecules, by passing the nanodroplet beam through a vapor pickup cell. The atoms or molecules quickly thermalize, in all their degrees of freedom, with the cold $\mathrm{He}$ nanoenvironment. They can recombine with other dopant atoms or molecules, if these are picked up by the same nanodroplet (the pick-up statistics is Poissonian); the binding energy is released into the He nano-bath. The ensuing species can then be sensitively probed via high-resolution spectroscopy. Atoms in He nanodroplets can thus form dimers (or larger oligomers).

The formation of cold heteronuclear alkali dimers NaK in helium droplets was previously observed by Higgins et al. $[107,108]$. In the present issue, the experimental paper by Mudrich, Bünerman, Stienkemeier, Dulieu and Weidemüller (pp. 291-299) reports the observation of the efficient formation of LiCs and NaCs in helium nanodroplets. The molecules are excited by a laser and then photo-ionized. The paper reports the excitation spectra recorded from mass selective photo-ionization. The potential curves correlating with the $\operatorname{Li}(2 s)+\operatorname{Cs}(5 d)$ and $\mathrm{Na}(3 s)+\mathrm{Cs}(5 d)$ asymptotic limits are obtained based on the measurement and compared with theoretical results from ab initio calculations with perturbative treatment of the fine structure. The discussion shows the need for a global frequency shift of the computed potentials. We note that little was known about the spectroscopy of LiCs and $\mathrm{NaCs}$ in the triplet state before this study was undertaken.

The authors point to the intriguing possibility of decelerating and trapping nanodroplets - along with the dopant molecules. This, they envision, could be achieved by first ionizing the nanodroplets (by electron impact or capture) and then applying a stopping potential. The cold and slow molecules could then be photo-desorbed and confined in an electrostatic trap. The flux of the moleculedoped He nanodroplets is estimated to be about $10^{10} \mathrm{~s}^{-1}$.

\section{Theory of cold collisions involving molecules}

The production of ultracold molecules has led chemists to ask the questions: What is the efficiency of chemical reactions and inelastic energy transfer in molecular collisions at low temperatures? Are chemical reactions possible at temperatures near absolute zero?

Based on the analysis of Landau and Lifshitz [109], Balakrishnan, Kharchenko, Forrey and Dalgarno showed that elastic and inelastic molecular scattering can be characterized in the limit of ultralow collision velocity by a complex scattering length [110]

$$
a_{n}=\alpha_{n}-i \beta_{n} .
$$

The imaginary part of the scattering length $\beta_{n}$ is directly proportional to the total cross-section for inelastic scattering in the state $n$

$$
\beta_{n}=k_{n} \sigma_{n}^{\text {in }} / 4 \pi,
$$

where $\sigma_{n}^{\text {in }}=\sum_{n^{\prime} \neq n} \sigma_{n \rightarrow n^{\prime}}, k_{n}=\mu v_{n} / \hbar, v_{n}$ is the collision velocity and $\mu$ is the reduced mass of the collision system. Equation (2) can be used to evaluate the zero temperature rate coefficient for inelastic energy transfer or chemical reaction

$$
R_{n}(T=0 \mathrm{~K})=4 \pi \beta_{n} \hbar / \mu .
$$

Because $\sigma_{n}^{\text {in }} \sim 1 / k_{n}$ according to the Wigner law, $\beta_{n}$ is constant in the limit $k_{n} \rightarrow 0$ and the rate coefficient (3) is finite at zero temperature.

The elastic cross-section is given by

$$
\sigma_{n \rightarrow n}=4 \pi\left(\alpha_{n}^{2}+\beta_{n}^{2}\right) .
$$

The positions and lifetimes of the bound levels of the collision complex lying just below dissociation threshold can be estimated from the real and imaginary parts of the scattering length as follows [110]:

$$
E=-\frac{\hbar^{2}}{2 \mu\left|a_{n}\right|}\left(\cos 2 \gamma_{n}+\mathrm{i} \sin 2 \gamma_{n}\right)=E_{n}-\mathrm{i} \Gamma_{n} / 2,
$$

where $\gamma_{n}=\arctan \left(\beta_{n} / \alpha_{n}\right)$. The energy $E$ is real for single channel scattering because $\beta_{n}=0$ and $\gamma_{n}=0$. When there is more than one open channel, the bound levels can decay through transitions into lower levels with a lifetime

$$
\tau_{n}=\hbar / \Gamma_{n}=\mu\left|a_{n}\right|^{4} /\left(2 \hbar \alpha_{n} \beta_{n}\right)
$$


that can be rewritten in terms of the zero energy elastic cross-section and zero temperature rate coefficient (3) as follows:

$$
\tau_{n}=\frac{\sigma_{n \rightarrow n}\left(k_{n} \rightarrow 0\right)\left|a_{n}\right|^{2}}{2 R_{n}(T=0) \alpha_{n}} .
$$

Forrey et al. [111] showed that the expression (7) can be used to estimate the positions and lifetimes of Feshbach resonances occurring in the incident channel in $s$-wave collisions. The derivation of equation (7) is based on multichannel effective-range theory and it can be applied only to the states that lie close to thresholds. More details on threshold phenomena in atom-atom, atom-molecule and electron-atom collisions can be found in a comprehensive review by Sadeghpour et al. [112], the review by Krems [113] and the paper of Mies and Raoult [114].

The Wigner law establishes that rate coefficients for inelastic collisions and chemical reactions at zero absolute temperature are finite. The magnitudes of the zero temperature rates for inelastic scattering of atoms and molecules were, however, not known until recently. The first calculations of rate constants for vibrational relaxation in atom-molecule collisions at ultralow temperatures were carried out by Schwenke and Truhlar [115]. They demonstrated that conventional quantum theory of vibrational relaxation is adequate for interpretation of low energy atom-molecule collisions and showed that low energy cross-sections follow the trends predicted by Wigner. The rate constants for the $\mathrm{He}-\mathrm{I}_{2}$ system studied by Schwenke and Truhlar were, however, extremely small in the zero temperature limit and it was concluded that the zero energy divergence of inelastic cross-sections may not affect the rate coefficients for inelastic scattering at temperatures $\geq 10^{-4} \mathrm{~K}$ in molecular systems. More recent calculations demonstrate that often this is not so.

The studies of the $\mathrm{He}+\mathrm{H}_{2}$ and other collision systems [116-128] showed that zero temperature rate coefficients for vibrational, rotational and electronic energy transfer in atom-atom and atom-molecule collisions may have substantial magnitudes. It was demonstrated by several authors that the attractive part of the interaction potential in the entrance collision channel is critical for the dynamics of ultracold scattering. Dashevskaya and Nikitin presented a simple explanation of this phenomenon using a generalized Landau-Lifshitz treatment of a collision problem [129].

The stability of rotationally hot molecules in a cold buffer gas has been explored in rigorous calculations of atom-molecule collisions at ultracold temperatures presented in this issue (Forrey pp. 409-415). Interesting implications for observation of ultracold super-rotors and quasiresonant energy transfer at very low collision energy should be appealing to experimental investigators.

Chemical reactions can be of two types: insertion reactions and abstraction reactions. The insertion reactions are often barrierless. Quantum mechanical calculations of chemical reactions are usually carried out in hyperspherical coordinates. Probabilities of chemical reactions are obtained from the solution of coupled differential equations on a grid of a single coordinate - the hyperradius.
The total wave function of the reactive complex may be expanded in terms of products of asymptotic functions corresponding to different reaction arrangements or in a basis of functions that vary with the hyperradius. The former procedure is suitable for the description of abstraction reactions, while the latter should be used for insertion reactions.

An example of an insertion reaction is the reaction of an alkali atom with an alkali diatomic molecule, like $\mathrm{Na}+\mathrm{Na}_{2}$. Soldán and coworkers used a hyperspherical coordinate quantum mechanical method of Launay and Le Dourneuf [130] to investigate the $\mathrm{Na}+\mathrm{Na}_{2}$ reaction at ultracold temperatures [131]. They showed that the crosssections for the reaction removing the vibrationally excited $\mathrm{Na}_{2}(v=1)$ molecules follow the Wigner law and the rate coefficient for the reaction is large in the limit of zero temperature. Recently, the same authors have extended their calculation to study the $\mathrm{Li}+\mathrm{Li}_{2}$ reaction at zero temperature [132]. It was shown that for low-lying bound states of $\mathrm{Li}_{2}$ there is no systematic difference between the inelastic collision rates for molecules formed from fermionic and bosonic Li.

The abstraction reactions have a potential barrier which separates the reactants from products. An example of an abstraction reaction is the chemical reaction of $\mathrm{F}$ with $\mathrm{H}_{2}$. Because the reaction barrier always has a large magnitude on the ultracold energy scale, it might be expected that the abstraction reactions would not occur at ultracold temperatures. Balakrishnan and Dalgarno presented the first investigation of an abstraction reaction at ultracold temperatures [133]. It was found that the cross-sections for the chemical reaction $\mathrm{F}+\mathrm{H}_{2} \rightarrow$ $\mathrm{FH}+\mathrm{H}$ follow the $s$-wave Wigner law and have surprisingly large magnitudes at ultralow energies. The rate coefficient for the reaction at zero temperature was calculated to be $1.25 \times 10^{-12} \mathrm{~cm}^{3} \mathrm{~s}^{-1}$. It was suggested that the large rate of the reaction at ultracold temperatures is due to tunneling of the light $\mathrm{H}$ atom under the reaction barrier. Several later studies of $\mathrm{F}+\mathrm{D}_{2}$ collisions [134-136] indicated that tunneling plays an important role in the reaction. A very recent article by Bodo, Gianturco, Balakrishnan and Dalgarno demonstrated that the chemical reaction of $\mathrm{F}$ with $\mathrm{H}_{2}$ at ultracold temperatures is assisted by a virtual resonance state near collision threshold [137]. A slight variation of the mass of $\mathrm{H}_{2}$ shifts the virtual level in and out of resonance with the collision energy, leading to dramatic enhancement or suppression of the reaction rate.

Two articles in this issue (Weck and Balakrishnan pp. 417-421 and Bodo and Gianturco pp. 423-427) report calculations of chemical reactions with large activation barriers in the limit of zero temperature. It is demonstrated that abstraction chemical reactions may be very fast at ultracold temperatures and the reactivity is enhanced to a great extent by internal excitation of the reactants. In particular, it is shown that the rate of the $\mathrm{F}+\mathrm{H}_{2}$ reaction is larger than the rate of collisional relaxation of the reactants when the $\mathrm{H}_{2}$ molecule is excited to an energy level above the activation barrier. These studies 
will potentially lead to the development of the field of ultracold chemistry as they show that chemical reactions do occur at zero absolute temperature. Studies of ultracold chemistry in external fields will open up the field of controlled chemistry.

The possibility of the creation of ultracold molecules is determined by their lifetime in an external field trap. The stability of cold and ultracold molecules in magnetic traps was studied in a series of papers by Bohn and coworkers [138-141] and Krems and coworkers [142-144]. Krems and Dalgarno proposed a theory for quantum mechanical calculations of molecular collisions in a magnetic trap and showed that trap loss of magnetically trapped diatomic molecules in electronic ${ }^{2} \Sigma$ or ${ }^{3} \Sigma$ states and in the rotational ground state is determined by the coupling to rotationally excited states [142]. The rotationally excited states are not energetically allowed at low collision energies so collision-induced spin relaxation occurs through virtual excitations in the reactive complex and it is slow. This study identified the range of molecules that may be amenable to magnetic trapping and evaporative cooling to ultracold temperatures. Novel states in dimers of ultracold molecules linked by an electric field were found by Bohn and coworkers [145].

To explore the prospects for sympathetic cooling of molecules by collisions with ultracold atoms, Soldán and Hutson computed highly accurate interaction potentials of the $\mathrm{Rb}-\mathrm{NH}$ complex in various spin states [146]. They showed that collision dynamics of ultracold $\mathrm{Rb}$ atoms with $\mathrm{NH}$ molecules may be complicated by the coupling to ionpair states leading to dramatic enhancement of the interaction strength.

Krems and Dalgarno derived threshold laws for reorientation of electronic angular momentum in atomic and molecular collisions in the absence of external fields [147]. Together with the computational study of Volpi and Bohn [141], this analysis indicates that the Zeeman relaxation rate in ultracold collisions of atoms or molecules should increase dramatically with increasing field strength.

An important discovery is reported in the article of this issue (Kłos, Rode, Rode, Chałasiński, and Szczęśniak pp. 429-437) describing interactions of non-S-state transition metal atoms with He. Non-S-state atoms interact upon collisions with He effectively like diatomic molecules $[148,149]$ and collisional cooling of non-S-state atoms in a magnetic trap has been predicted to be impossible [150, 151]. The study of Kłos and coworkers, however, demonstrates that the angular dependence of the interaction potential between transition metal atoms and $\mathrm{He}$ is dramatically suppressed. This indicates that transition metal atoms should be amenable to buffer-gas loading in a magnetic trap and, possibly, evaporative cooling to ultracold temperatures. Trapping of non-S-state atoms will greatly expand the scope of ultracold molecular physics. Photoassociation of ultracold non-S-state atoms will produce ultracold non- $\Sigma$-state molecules.

Giovanazzi and O'Dell present in this issue (pp. 439445) a discussion of Bose-Einstein condensates of species with strong dipolar interactions in a quasi-1D geometry.
The authors suggest a method for stabilizing such a system, by reversing the sign of the dipolar interaction. They find a roton minimum in the spectrum of Bogolyubov excitations and suggest the possibility of tuning the depth of this minimum. The authors point out that by changing the minimum depth, it may become possible to induce the transition from a homogeneous superfluid to a chargedensity wave.

\section{Conclusions and future directions}

We hope that the readers of this issue will be convinced of the rapid expansion of the field of ultracold molecules. We should note the combination of traditional physics (accurate molecular spectroscopy) and very recent research advances (molecular quantum gases). We should also note the strong interplay between theory and experiment, which appears in many papers of this issue. We expect the next few years will be marked by the development of improved techniques for the production of ultracold molecules, and by more fundamental studies with cold and ultracold molecules as model systems and as a tool for very precise measurements.

It is clear from the present series of papers that there are many roads to cold molecules. The groups working with indirect methods like photoassociation of ultracold atoms will develop efficient ways of creating molecules in the vibrational ground level, making use of various technologies (e.g., Raman schemes, chirped pulses) that have yet to be fully studied. The groups working with direct methods will develop techniques to reach lower temperatures. In either case, trapping molecules and detecting them are key issues.

The development of new types of traps for molecules in high-field seeking states will likely be pursued as a priority by several groups. Apart from the microwave trap proposed in this issue, it is the ac trap for polar - and perhaps even paramagnetic - molecules that raises great expectations. Such traps could confine ground state molecules, which are immune to dipolar relaxation and other loss processes. This would greatly improve the chances for achieving evaporative cooling - and reaching the ultracold regime. Such traps offer a large volume and might be loaded by optical pumping of slow molecules in suitable excited states. The presence of the trapping field is expected to greatly enhance the scattering cross-section by aligning the molecular dipoles, and thus reduce the requisite number density of the trapped molecules. Alternatively, the critical density conducive to evaporation may be achieved by bunching. Evaporative cooling is also likely to be attained in magnetic traps, which can be loaded with large numbers of molecules using the beam-loaded buffer gas technique. Another possibility is to use a hydride molecule plus laser cooling to directly load the molecules into a magnetic, electrostatic or ac trap. Sympathetic cooling of molecules (or molecular ions) with laser cooled atoms (or atomic ions) is also being pursued in a number of laboratories. 
Cooling Rydberg molecules is an interesting challenge to explore [92-94].

During the next few years, new experiments are likely to be carried out that will make use of cold molecules. Scattering of slow molecules by atoms confined in a MOT, or of successive bunches in a decelerator, storage ring or trap by one another are among the pursuits likely to be taken up in several laboratories. The implementation of a molecular fountain is a prominent goal.

Chemical reactions have been shown to occur rapidly at temperatures near zero Kelvin and further studies, both experimental and theoretical, will demonstrate the uniqueness of ultracold chemistry. It may be expected that selection rules are more pronounced and branching ratios of chemical reactions enhanced at ultralow temperatures [152]. The study of ultracold chemistry will take us into a strange new world. Even the smallest activation energy will surely exceed the available thermal energy. However, the large de Broglie wavelength pertaining to the ultracold regime entirely changes the nature of reaction dynamics. At such low temperatures, even the collisions of large molecules exhibit significant quantum effects. Energy barriers on the potential energy surface play a different role because, in the hyperquantum regime, tunneling becomes the dominant reaction pathway. Since tunneling and resonances are characteristic of this regime, they can serve as ultra-sensitive probes of particular features of the potential energy surface.

Interactions of molecules at low temperatures can be tuned by external electromagnetic fields and the creation of cold and ultracold molecules may lead to the development of the field of controlled chemistry. External-field control of chemical reactions can be based on several principles. The Zeeman and Stark effects may remove some of the energetically allowed reaction paths or they may open closed reaction channels [153], leading to suppression or enhancement of the reaction efficiency. External fields couple the states of different total angular momenta, so that forbidden electronic transitions may become allowed in an external field and the transition rate may be controlled by the field strength [150]. As the $\mathrm{F}+\mathrm{H}_{2}$ reaction shows, the rate of low temperature abstraction reactions may be dramatically enhanced by the presence of a resonance state near threshold. By shifting molecular energy levels with external fields, it should be possible to bring an excited bound level of the reactive complex in resonance with the collision energy [137]. External fields may influence kinetic properties of ultracold molecular gases such as diffusion.

Achieving quantum degeneracy in an ultracold ensemble of polar molecules will greatly expand the scope for study and applications of collective quantum phenomena in this new form of matter. Of primary interest are the effects of rotational and vibrational excitation and their manifestation in collisional properties. In particular, a BEC of polar molecules would represent a system of relatively strongly interacting particles. This could be used in elucidating the link between BECs in gases and in liquids. The study of ultracold fermionic molecules is also of great interest: the electric dipole-dipole interaction, which is predicted to be energy independent, may give rise to a molecular superfluid via BCS pairing. This may take place at phase space parameters achievable by evaporative cooling. Ultracold molecules could be also used in quantum computation. The scheme invokes ultracold ground-state molecules trapped in a one-dimensional optical lattice combined with an inhomogeneous electrostatic field [24].

There is also a class of experiments with cold molecules that could answer questions reaching beyond chemistry and molecular physics: these experiments test fundamental symmetries in nature, such as the time-reversal symmetry and the symmetrization postulate. A possible violation of time reversal symmetry may be revealed by measuring the electric dipole moment (EDM) of the electron in heavy diatomic molecules. It is particularly thrilling that the current experimental upper bound on EDM is close to several predictions made by theories that go beyond the Standard Model [154-160]. An increase in accuracy by even one order of magnitude would therefore have a dramatic impact on theory. Also rapid progress in testing the Pauli principle for bosons with cold molecules (with zerospin nuclei) is expected. Another fundamental experiment tests parity violation, via high-resolution spectroscopy of enantiomers of chiral molecules [161]. The accuracy of this experiment is expected to be significantly enhanced by using cold molecules.

We acknowledge the Institute for Theoretical Atomic, Molecular and Optical Physics (ITAMP) at Harvard University and the Smithsonian Astrophysical Observatory and the Center for Ultracold Atoms (CUA) at Harvard University and Massachusetts Institute of Technology for supporting the 2004 Workshop on ultracold polar molecules. We thank John Bohn for his help on the Feshbach resonance section and Kate Kirby for useful comments on the manuscript. We thank Franco Gianturco for encouraging the idea to organize this issue and the European Physical Journal D for the opportunity to publish this sizable collection of papers.

\section{References}

1. M. Di Rosa, ITAMP-CUA Workshop on Ultracold Polar Molecules, Cambridge, 2004

2. J.T. Bahns, W.C. Stwalley, P.L. Gould, Laser cooling of molecules: a sequential scheme for rotation, translation, and vibration, J. Chem. Phys. 104, 9689 (1996)

3. J. Weinstein, R. deCarvalho, T. Guillet, B. Friedrich, J.M. Doyle, Magnetic trapping of calcium monohydride molecules at millikelvin temperatures, Nature 395, 148 (1998)

4. C.A. Stan, M.W. Zwierlein, C.H. Schunck, S.M.F. Raupach, W. Ketterle, Interspecies Feshbach Resonances, preprint at www.arXiv.org/cond-mat/0406129 (2004)

5. S. Inouye, J. Goldwin, M.L. Olsen, C. Ticknor, J.L. Bohn, D.S. Jin, Observation of Heteronuclear Feshbach Resonances in a Bose-Fermi Mixture, preprint at www.arXiv.org/cond-mat/0406208 (2004)

6. D. Wang, J. Qi, M.F. Stone, O. Nikolayeva, H. Wang, B. Hattaway, S.D. Gensemer, P.L. Gould, E.E. Eyler, W.C. 
Stwalley, Photoassociative production and trapping of ultracold KRb molecules, Phys. Rev. Lett. (in press, 2004)

7. A.J. Kerman, J.M. Sage, S. Sainis, T. Bergeman, D. DeMille, Production and state-selective detection of ultracold, ground state RbCs molecules, preprint at www.arXiv.org/physics/0402116m (2004)

8. M.W. Mancini, G.D. Telles, A.R.L. Caires, V.S. Bagnato, L.G. Marcassa, Observation of ultracold ground-State heteronuclear molecules, Phys. Rev. Lett. 92, 133203 (2004)

9. C. Haimberger, J. Kleinert, M. Bhattacharya, N.P. Bigelow, Formation and detection of ultracold groundstate polar molecules, Phys. Rev. A 70, 21402 (2004)

10. C.L. Cesar, D.M. Silveira, M.G. Veloso, F. Zappa, C.C. Rodegheri, "Buffer gas trap for hydrogen spectroscopy", in Hydrogen Atom III, The international conference on Precision Physics of Simple Atomic Systems, edited by C.L. Cesar, S.G. Karshenboim, V.A. Shelyuto (2004)

11. A. Peters, "A cryogenic magnetic trap for ultracold atoms and molecules", in Quantum Gases International Workshop, p. 19 (2003)

12. D. DeMille, private communication (2004)

13. R. deCarvalho, N. Brahms, B. Newman, C. Johnson, L. Willman, J.M. Doyle, T.J. Greytak, D. Kleppner, "A New Path to Ultracold Hydrogen", in Hydrogen Atom III, The international conference on Precision Physics of Simple Atomic Systems, edited by C.L. Cesar, S.G. Karshenboim, V.A. Shelyuto (2004)

14. H.L. Bethlem, G. Meijer, Production and application of translationally cold molecules, Int. Rev. Phys. Chem. 22, 73 (2003)

15. J.R. Bochinski, E.R. Hudson, H.J. Lewandowski, J. Ye, Cold free radical molecules in the laboratory frame, Phys. Rev. A (submitted)

16. E.A. Hinds, Testing time reversal symmetry using molecules, Phys. Scripta T70, 34 (1997)

17. M.R. Tarbutt, H.L. Bethlem, J.J. Hudson, V.L. Ryabov, V.A. Ryzhov, B.E. Sauer, G. Meijer, E.A. Hinds, Slowing heavy, ground-state molecules using an alternating gradient decelerator, Phys. Rev. Lett. 92, 173002 (2004)

18. H. Gould, Department of Energy, Basic Energy Science Contractor's Meeting, 2003.

19. P.F. Barker, A.I. Bishop, R. Fulton, M.N. Shneider, Slowing molecules with pulsed optical lattices, in $A C S$ 2004 National Meeting Technical Program (2004)

20. M. Gupta, D. Herschbach, A mechanical means to produce intense beams of slow molecules, J. Phys. Chem. A 103, 10670 (1999)

21. M.S. Elioff, J.J. Valentini, D.W. Chandler, Subkelvin cooling NO molecules via billiard-like collisions with argon, Science 302, 1940 (2003)

22. S.A. Rangwala, T. Junglen, T. Rieger, P.W.H. Pinkse, G. Rempe, Continuous source of translationally cold dipolar molecules, Phys. Rev. A 67, 043406 (2003)

23. E. Nikitin, E. Dashevskaya, J. Alnis, M. Auzinsh, E.R.I. Abraham, B.R. Furneaux, M. Keil, C. McRaven, N. Shafer-Ray, R. Waskowsky, Measurement and prediction of the speed-dependent throughput of a magnetic octupole velocity filter including nonadiabatic effects, Phys. Rev. A 68, 023403 (2003)

24. D. DeMille, Quantum computation with trapped polar molecules, Phys. Rev. Lett. 88, 067901 (2002)

25. L. Santos, G.V. Shlyapnikov, P. Zoller, M. Lewenstein, Bose-Einstein Condensation in Trapped Dipolar Gases, Phys. Rev. Lett. 85, 1791 (2000)
26. M. Baranov, L. Dobrek, K. Goral, L. Santos, M. Lewenstein, Ultracold dipolar gases - a challenge for experiments and theory, Phys. Scripta T102, 74 (2002)

27. L.D. Carr, G.V. Shlyapnikov, Y. Castin, Achieving a BCS Transition in an Atomic Fermi Gas, Phys. Rev. Lett. 92, 150404 (2004)

28. M.A. Baranov, Ł. Dobrek, M. Lewenstein, Superfluidity of trapped dipolar Fermi Gases, Phys. Rev. Lett. 92, 250403 (2004)

29. D. Kleppner, Professor Feshbach and his resonance, Phys. Today 57, 12 (2004)

30. N. Djeu, W.T. Whitney, Laser cooling by spontaneous anti-Stokes scattering, Phys. Rev. Lett. 46, 238 (1981)

31. H.R. Thorsheim, J. Weiner, P.S. Julienne, Laser-induced photoassociation of ultracold sodium atoms, Phys. Rev. Lett. 58, 2420 (1987)

32. J. Weiner, V.S. Bagnato, S.C. Zilio, P.S. Julienne, Experiments and theory in cold and ultracold collisions, Rev. Mod Phys. 71, 1 (1999)

33. J.T. Bahns, W.C. Stwalley, P.L. Gould, Formation of cold $(T \leq 1 K)$ molecules, Adv. At. Mol. Opt. Phys. 42, 171 $(2000)$

34. F. Masnou-Seeuws, P. Pillet, Formation of ultracold molecules $(T<200 \mu K)$ via photoassociation in a gas of laser-cooled atoms, Adv. At. Mol. Opt. Phys. 47, 53 (2001)

35. W.C. Stwalley, H. Wang, Special review lecture Photoassociation of ultracold atoms: A new spectroscopic technique, J. Mol. Spectrosc. 195, 194 (1999)

36. T. Takekoshi, B.M. Patterson, R.J. Knize, Observation of optically trapped cold cesium molecules, Phys. Rev. Lett. 81, 5105 (1999)

37. A. Fioretti, D. Comparat, A. Crubellier, O. Dulieu, F. Masnou-Seeuws, P. Pillet, Formation of cold $\mathrm{Cs}_{2}$ molecules through photoassociation, Phys. Rev. Lett. 80, 4402 (1998)

38. T. Takekoshi, B.M. Patterson, R.J. Knize, Observation of cold ground-state cesium molecules produced in a magneto-optical trap, Phys. Rev. A 59, R5 (1998)

39. N. Nikolov, E.E. Eyler, X.T. Wang, J. Li, H. Wang, W.C. Stwalley, P. Gould, Observation of translationally ultracold ground state potassium molecules, Phys. Rev. Lett. 82, 703 (1999)

40. A.N. Nikolov, J.R. Ensher, E.E. Eyler, H. Wang, W.C. Stwalley, P. Gould, Efficient Production of Ground-State Potassium Molecules at Sub-mK Temperatures by TwoStep Photoassociation, Phys. Rev. Lett. 84, 246 (2000)

41. C. Gabbanini, A. Fioretti, A. Lucchesini, S. Gozzini, M. Mazzoni, Observation of translationally cold ground state rubidium molecules, Phys. Rev. Lett. 84, 2814 (2000)

42. C.M. Dion, C. Drag, O. Dulieu, B. Laburthe Tolra, F. Masnou-Seeuws, P. Pillet, Resonant coupling in the formation of ultracold ground state molecules via photoassociation, Phys. Rev. Lett. 86, 2253 (2001)

43. F. Fatemi, K. Jones, P. Lett, E. Tiesinga, Ultracold ground-state molecule production in sodium, Phys. Rev. A 66, 053401 (2002)

44. H. Wang, W.C. Stwalley, Ultracold photoassociative spectroscopy of heteronuclear alkali-metal diatomic molecules, J. Chem. Phys. 108, 5767 (1998)

45. J.P. Shaffer, W. Chalupezak, N.P. Bigelow, Photoassociative ionization of heteronuclear molecules in a novel two-species magneto-optical trap, Phys. Rev. Lett. 82, 1124 (1999) 
46. U. Schloder, H. Engler, U. Schunemann, R. Grimm, M. Weidemuller, Cold inelastic collisions between lithium and cesium in a two species magneto-optical trap, Eur. Phys. J. D 7, 331 (1999)

47. A.J. Kerman, J.M. Sage, S. Sainis, T. Bergeman, D. DeMille, Production of Ultracold, Polar RbCs* Molecules via Photoassociation, Phys. Rev. Lett. 92, 033004 (2004)

48. A.J. Kerman, J.M. Sage, S. Sainis, T. Bergeman, D. DeMille, Photoassociation and State-Selective Detection of Ultracold RbCs Molecules, Phys. Rev. Lett. 92, 153001 (2004)

49. M.W. Mancini, G.D. Telles, A.R.L. Caires, V.S. Bagnato, L.G. Marcassa, Observation of ultracold ground-state heteronuclear molecules, Phys. Rev. Lett. 92, 133203 (2004)

50. N. Vanhaecke, W. DeSouza Melo, B. Laburthe Tolra, D. Comparat, P. Pillet, Accumulation of cold cesium molecules via photoassociation in a mixed atomic and molecular trap, Phys. Rev. Lett. 89, 063001 (2002)

51. D.J. Heinzen, unpublished; R.S. Freeland, Ph.D. thesis, University of Texas, pp. 112-122

52. W.T. Zemke, W.C. Stwalley, Radiative transition probabilities, lifetimes and dipole moments for the vibrational levels of the $X^{1} \Sigma^{+}$ground state of ${ }^{39} K^{85} R b$, J. Chem. Phys. 120, 88 (2004)

53. H. Wang, X.T. Wang, P.L. Gould, W.C. Stwalley, Opticaloptical double resonance photoassociative spectroscopy of ultracold ${ }^{39} \mathrm{~K}$ atoms near highly excited asymptotes, Phys. Rev. Lett. 78, 4173 (1997)

54. M.L. Almazor, O. Dulieu, M. Elbs, E. Tiemann, F. Masnou-Seeuws, How to get access to long range states of highly excited molecules, Eur. Phys. J. D 5, 237 (1999)

55. T. Laue, P. Pellegrini, O. Dulieu, C. Samuelis, H. Knoeckel, F. Masnou-Seeuws, E. Tiemann, Observation of the long-range potential well of the $(6)^{1} \Sigma_{g}^{+}$state of $\mathrm{Na}_{2}$, Eur. Phys. J. D 26, 173 (2003)

56. J. Léonard, M. Walhout, A. Mosk, T. Müller, M. Leduc, C. Cohen-Tannoudji, Giant helium dimers produced by photoassociation of ultracold metastable atoms, Phys. Rev. Lett. 91, 073203 (2003)

57. E. Luc-Koenig, R. Kosloff, F. Masnou-Seeuws, M. Vatasescu, Time-dependent analysis of photoassociation of cold cesium atoms using chirped laser pulses, Phys. Rev. A 70, (2004)

58. C. Koch, J. Palao, R. Kosloff, F. Masnou-Seeuws, How to obtain ultracold $v=0$ molecules using optimal control theory, Phys. Rev. A 70, 013402 (2004)

59. P. Fedichev, Y. Kagan, G.V. Shlyapnikov, J. Walraven, Influence of nearly resonant light on the scattering length in low-temperature atomic gases, Phys. Rev. Lett. 77, 2913 (1996)

60. J.L. Bohn, P.S. Julienne, Prospects for influencing scattering lengths with far-off resonant light, Phys. Rev. A 56, 1486 (1997)

61. V. Kokoouline, J. Vala, R. Kosloff, Tuning the scattering length on the ground triplet state of $\mathrm{Cs}_{2}$, J. Chem. Phys. 114, 3046 (2001)

62. F.K. Fatemi, K.M. Jones, P.D. Lett, Observation of optically induced Feshbach resonances in collisions of cold atoms, Phys. Rev. Lett. 85, 4462 (2000)

63. M. Theis, G. Thalhammer, K. Winkler, M. Hellwig, G. Ruff, R. Grimm, J.H. Denschlag, Tuning the scattering length with an optically induced Feshbach resonance, Phys. Rev. Lett. 93, 123001 (2004)
64. W.C. Stwalley, Stability of spin-aligned hydrogen at low temperatures and high magnetic fields: new fielddependent scattering resonances and predissociations, Phys. Rev. Lett. 37, 1628 (1976)

65. E. Tiesinga, A.J. Moerdijk, B.J. Verhaar, H.T.C. Stoof, Conditions for Bose-Einstein condensation in magnetically trapped atomic cesium, Phys. Rev. A 46, R1167 (1992)

66. E. Tiesinga, B.J. Verhaar, H.T.C. Stoof, Threshold and resonance phenomena in ultracold ground-state collisions, Phys. Rev. A 47, 4114 (1993)

67. P.S. Julienne, E. Tiesinga, T. Koehler, Making cold molecules by time-dependent Feshbach resonances, J. Mod. Opt. 51, 1787 (2004)

68. D.S. Petrov, C. Salomon, G.V. Shlyapnikov, Weakly bound dimers of Fermionic atoms, Phys. Rev. Lett. 93, 090404 (2004)

69. D.S. Petrov, Three-body problem in Fermi gases with short-range interparticle interaction, Phys. Rev. A 67, 010703(R) (2003)

70. D.S. Petrov, C. Salomon, G.V. Shlyapnikov, Scattering properties of weakly bound dimers of fermionic atoms, Phys. Rev. A (in press, 2004)

71. S. Inouye, M.R. Andrews, J. Stenger, H.J. Miesner, D.M. Stamper-Kurn, W. Ketterle, Observation of Feshbach resonances in a Bose-Einstein condensate, Nature 392, 151 (1998)

72. Ph. Courteille, R.S. Freeland, D.J. Heinzen, A. van Abeelen, B.J. Verhaar, Observation of a Feshbach resonance in cold atom scattering, Phys. Rev. Lett. 81, 69 (1998)

73. J.L. Roberts, N.R. Claussen, J.P. Burke Jr, C.H. Greene, E.A. Cornell, C.E. Wieman, Resonant magnetic field control of elastic scattering in cold ${ }^{85} R b$, Phys. Rev. Lett. 81, 5109 (1998)

74. C. Chin, V. Vuletic, A.J. Kerman, S. Chu, High resolution Feshbach spectroscopy of cesium, Phys. Rev. Lett. 85, 2717 (2000)

75. A. Marte, T. Volz, J. Schuster, S. Dürr, G. Rempe, E.G.M. van Kempen, B.J. Verhaar, Feshbach resonances in rubidium 8\%: precision measurement and analysis, Phys. Rev. Lett. 89, 283202 (2002)

76. E.A. Donley, N.R. Claussen, S.T. Thompson, C.E. Wieman, Atom-molecule coherence in a Bose-Einstein condensate, Nature 417, 529 (2002)

77. M. Greiner, C.A. Regal, D.S. Jin, Emergence of a molecular Bose-Einstein condensate from a Fermi gas, Nature 426, 537 (2003)

78. S. Jochim, M. Bartenstein, A. Altmeyer, G. Hendl, S. Riedl, C. Chin, J. Hecker Denschlag, R. Grimm, BoseEinstein condensation of molecules, Science 302, 2101 (2003)

79. M.W. Zwierlein, C.A. Stan, C.H. Schunck, S.M.F. Raupach, S. Gupta, Z. Hadzibabic, W. Ketterle, Observation of Bose-Einstein condensation of molecules, Phys. Rev. Lett. 91, 250400 (2003)

80. T. Bourdel, L. Khaykovich, J. Cubizolles, J. Zhang, F. Chevy, M. Teichmann, L. Tarruell, S.J.J.M.F. Kokkelmans, C. Salomon, Experimental study of the $B E C-B C S$ crossover region in lithium 6, Phys. Rev. Lett. 93, $050401(2004)$

81. J. Kim, B. Friedrich, D.P. Katz, D. Patterson, J.D. Weinstein, R. deCarvalho, J.M. Doyle, Buffer-gas loading 
and magnetic trapping of atomic europium, Phys. Rev. Lett. 78, 3665 (1997)

82. R. deCarvalho, J.M. Doyle, B. Friedrich, T. Guillet, J. Kim, D. Patterson, J. Weinstein, Buffer-gas loaded magnetic traps for atoms and molecules: A primer, Eur. Phys. J. D 7, 289 (1999)

83. D.R. Willey, D.N. Bittner, F.C. Delucia, Collisional cooling of the NO-He system - the pressure broadening crosssections between 4.3 and 1.8 K, Mol. Phys. 67, 455 (1989)

84. H.L. Bethlem, G. Berden, G. Meijer, Decelerating neutral dipolar molecules, Phys. Rev. Lett. 83, 1558 (1999)

85. V. Veksler, J. Phys. (USSR) 9, 153 (1944)

86. E.M. McMillan, The Synchrotron Proposed High Energy Particle Accelerator, Phys. Rev. 68, 143 (1945)

87. H.L. Bethlem, F.M.H. Crompvoets, G. Berden, R.T. Jongma, S.Y.T. Meerakker, G. Meijer, Deceleration and trapping of ammonia using time-varying electric fields, Phys. Rev. A 65, 053416 (2002)

88. H.L. Bethlem, G. Berden, F.M.H. Crompvoets, R.T. Jongma, A.J.A. van Roij, G. Meijer, Electrostatic trapping of ammonia molecules, Nature 406, 491 (2000)

89. F.M.H. Crompvoets, H.L. Bethlem, R.T. Jongma, G. Meijer, A prototype storage ring for neutral molecules, Nature 411, 174 (2001)

90. J.A. Maddi, T.P. Dinneen, H. Gould, Slowing and cooling molecules and neutral atoms by time-varying electric-field gradients, Phys. Rev. A 60, 3882 (1999)

91. J.R. Bochinski, E.R. Hudson, H.J. Lewandowski, G. Meijer, J. Ye, Phase space manipulation of cold free radical OH molecules, Phys. Rev. Lett. 91, 243001 (2003)

92. S.R. Procter, Y. Yamakita, F. Merkt, T.P. Softley, Controlling the motion of hydrogen molecules, Chem. Phys. Lett. 374, 667 (2003)

93. E. Vliegen, H.J. Woerner, T.P. Softley, F. Merkt, Nonhydrogenic effects in the deceleration of Rydberg atoms in inhomogeneous electric fields, Phys. Rev. Lett. 92, 033005 (2004)

94. N. Vanhaecke, D. Comparat, P. Pillet, Rydberg deceleration using a travelling electric-field gradient, J. Phys. B: At. Mol. Opt. Phys. 37, 1 (2004)

95. H.L. Bethlem, G. Berden, A.J.A. van Roij, F.M.H. Crompvoets, G. Meijer, Trapping neutral molecules in a traveling potential well, Phys. Rev. Lett. 84, 5744 (2000)

96. W. Ubachs, E. Reinhold, Highly accurate $\mathrm{H}_{2}$ Lyman and Werner band laboratory measurements and an improved constraint on a cosmological variation of the proton-toelectron mass ratio, Phys. Rev. Lett. 92, 101302 (2004)

97. D. Auerbach, E.E.A. Bromberg, L. Wharton, AlternateGradient focusing of molecular beams, J. Chem. Phys. 45, 2160 (1966)

98. E. Peik, Alternate-gradient focusing of molecular beams, Eur. Phys. J. D 6, 179 (1999)

99. J.D. Miller, R.A. Cline, D.J. Heinzen, Far-off-resonance optical trapping of atoms, Phys. Rev. A 47, R4567 (1993)

100. S.Y.T. van de Meerakker, R.T. Jongma, H.L. Bethlem, G. Meijer, Accumulating NH radicals in a magnetic trap, Phys. Rev. A 64, 041401 (2001)

101. S. Goyal, D.L. Schutt, G. Scoles, Vibrational spectroscopy of sulfur hexafluoride attached to helium clusters, Phys. Rev. Lett. 69, 933 (1992)

102. F. Dong, R.E. Miller, Vibrational Transition Moment Directions for Determining the Structure of Isolated Biomolecules, Science 298, 1227 (2002)
103. R. Lehnig, A. Slenczka, Emission Spectra of Free Base Phthalocyanine in Superfluid Helium Droplets, J. Chem. Phys. 118, 8256 (2003)

104. S. Grebenev, J.P. Toennies, A.F. Vilesov, Superfluidity within a small helium-4 cluster: The microscopic Andronikashvili experiment, Science 279, 2083 (1998)

105. J.P. Toennies, A.F. Vilesov, K.B. Whaley, Superfluid helium droplets: an ultracold nanolaboratory, Phys. Today 54, 31 (2001)

106. C.J. Burnham, S.S. Xantheas, M.A. Miller, B.E. Applegate, R.E. Miller, The Formation of Cyclic Water Complexes by Sequential Ring Insertion: Experiment and Theory, J. Chem. Phys. 117, 1109 (2002)

107. J. Higgins, C. Callegari, J. Reho, F. Stienkemeier, W.E. Ernst, M. Gutowski, G. Scoles, Helium cluster isolation spectroscopy of alkali dimers in the triplet manifold, J. Phys. Chem. A 102, 4952 (1998)

108. J. Higgins, J. Reho, F. Stienkemeier, W.E. Ernst, K.K. Lehmann, G. Scoles, Spectroscopy in, on and off a beam of superfluid helium nanodroplets, Atomic and Molecular Beams: The State of the Art 2000, edited by R. Campargue (Springer-Verlag, 2000)

109. L.D. Landau, E.M. Lifshitz, Quantum mechanics (Pergamon Press, 1965)

110. N. Balakrishnan, V. Kharchenko, R.C. Forrey, A. Dalgarno, Complex scattering lengths in multi-channel atom-molecule collisions, Chem. Phys. Lett. 280, 5 (1997)

111. R.C. Forrey, N. Balakrishnan, V. Kharchenko, A. Dalgarno, Feshbach resonances in ultracold atom-diatom scattering, Phys. Rev. A 58, R2645 (1998)

112. H.R. Sadeghpour, J.L. Bohn, M.J. Cavagnero, B.D. Esry, I.I. Fabrikant, J.H. Macek, A.R.P. Rau, Collisions near threshold in atomic and molecular physics, J. Phys. B: At. Mol. Opt. Phys. 33, R93 (2000)

113. R.V. Krems, "Chemical reactions and inelastic collisions of atoms and molecules at cold and ultracold temperatures", in Recent Research Developments in Chemical Physics, Vol. 3, part II, pp. 485-500 (2002), ISBN: 817895-066-9.

114. F.H. Mies, M. Raoult, Analysis of threshold effects in ultracold atomic collisions, Phys. Rev. A 62, 012708 (2000)

115. D.W. Schwenke, D.G. Truhlar, The effect of Wigner singularities on low-temperature vibrational relaxation rates, J. Chem. Phys. 83, 3454 (1985)

116. N. Balakrishnan, R.C. Forrey, A. Dalgarno, Quenching of $\mathrm{H}_{2}$ vibrations in ultracold ${ }^{3} \mathrm{He}$ and ${ }^{4} \mathrm{He}$ collisions, Phys. Rev. Lett. 80, 3224 (1998)

117. N. Balakrishnan, A. Dalgarno, R.C. Forrey, Vibrational relaxation of $\mathrm{CO}$ by collisions with ${ }^{4} \mathrm{He}$ at ultracold temperatures, J. Chem. Phys. 113, 621 (2000)

118. C. Zhu, N. Balakrishnan, A. Dalgarno, Vibrational relaxation of $\mathrm{CO}$ in ultracold ${ }^{3}$ He collisions, J. Chem. Phys. 115, 1335 (2001)

119. N. Balakrishnan, A. Dalgarno, On the quenching of rovibrationally excited molecular oxygen at ultracold temperatures, J. Phys. Chem. A 105, 2348 (2001)

120. R.C. Forrey, N. Balakrishnan, A. Dalgarno, M.R. Haggerty, E.J. Heller, Quasiresonant energy transfer in ultracold atom-diatom collisions, Phys. Rev. Lett. 82, 2657 (1999)

121. J.C. Flasher, R.C. Forrey, Cold collisions between argon atoms and hydrogen molecules, Phys. Rev. A 65, 032710 (2002) 
122. E. Bodo, F.A. Gianturco, A. Dalgarno, Quenching of vibrationally excited $\mathrm{CO}(\mathrm{nu}=\mathrm{2})$ molecules by ultra-cold collisions with He-4 atoms, Chem. Phys. Lett. 353, 127 (2002)

123. R.V. Krems, A.A. Buchachenko, Ultracold inelastic atomic collisions: Threshold relaxation of $O\left({ }^{3} P_{0}\right)$ by $\mathrm{He}$, Phys. Rev. A 64, 024704 (2001)

124. R.V. Krems, A. Dalgarno, Shape resonances and nonadiabatic dynamics in $O\left({ }^{3} P_{j}\right)+$ He collisions at cold and ultracold temperatures, Phys. Rev. A 66, 012702 (2002)

125. P.E. Siska, Cold and ultracold ion-neutral inelastic collisions: Spin-orbit relaxation in $\mathrm{He}+\mathrm{Ne}^{+}$, J. Chem. Phys. 115, 4527 (2001)

126. R. Krems, A. Dalgarno, Electronic and rotational energy transfer in $\left.\mathrm{F}^{2} \mathrm{P}_{1 / 2}\right)+\mathrm{H}_{2}$ collisions at ultracold temperatures, J. Chem. Phys. 117, 118 (2002)

127. P. Florian, M. Hoster, R.C. Forrey, Rotational relaxation in ultracold $\mathrm{CO}+\mathrm{He}$ collisions, Phys. Rev. A 70, 032709 (2004)

128. K. Tilford, M. Hoster, P.M. Florian, R.C. Forrey, Cold collisions involving rotationally hot oxygen molecules, Phys. Rev. A 69, 052705 (2004)

129. E.I. Dashevskaya, E.E. Nikitin, Quantum suppression and enhancement of the quasiclassical Landau-Lifshitz matrix elements: Application to the inelastic $\mathrm{H}_{2}$-He scattering at ultralow energies, Phys. Rev. A 63, 012711 (2000)

130. J.-M. Launay, M. Le Dourneuf, Hyperspherical closecoupling calculation of integral cross-sections for the reaction $\mathrm{H}+\mathrm{H}_{2} \rightarrow \mathrm{H}_{2}+\mathrm{H}$, Chem. Phys. Lett. 163, 178 (1989)

131. P. Soldán, M.T. Cvitaš, J.M. Hutson, P. Honvault, J.-M. Launay, Quantum dynamics of ultracold $\mathrm{Na}+\mathrm{Na}_{2}$ collisions, Phys. Rev. Lett. 89, 153201 (2002)

132. M.T. Cvitaš, P. Soldá, J.M. Hutson, P. Honvault, J.-M. Launay, Ultracold $\mathrm{Li}+\mathrm{Li}_{2}$ collisions: bosonic and fermionic cases, preprint at www. arXiv.org/cond-mat/0409709

133. N. Balakrishnan, A. Dalgarno, Chemistry at ultracold temperatures, Chem. Phys. Lett. 341, 652 (2001)

134. E. Bodo, F.A. Gianturco, A. Dalgarno, The reaction of $\mathrm{F}+\mathrm{D}_{2}$ at ultra-low temperatures: the effect of rotational excitation, J. Phys. B: At. Mol. Opt. Phys. 35, 2391 (2002)

135. E. Bodo, F.A. Gianturco, A. Dalgarno, $F+D_{2}$ reaction at ultracold temperatures, J. Chem. Phys. 116, 9222 (2002)

136. N. Balakrishnan, A. Dalgarno, J. Phys. Chem. A 107, 7101 (2003)

137. E. Bodo, F.A. Gianturco, N. Balakrishnan, A. Dalgarno, Chemical reaction in the limit of zero kinetic energy: virtual states and Ramsauer minima in $\mathrm{F}+\mathrm{H}_{2} \rightarrow \mathrm{HF}+\mathrm{H}$, J. Phys. B: At. Mol. Opt. Phys. 37, 3641 (2004)

138. J.L. Bohn, Molecular spin relaxation in cold atommolecule scattering, Phys. Rev. A 61, 040702(R) (2000)

139. J.L. Bohn, Cold collisions of $\mathrm{O}_{2}$ with helium, Phys. Rev. A 62, $032701(2000)$

140. A.V. Avdeenkov, J.L. Bohn, Ultracold collisions of oxygen molecules, Phys. Rev. A 64, 052703 (2001)

141. A. Volpi, J.L. Bohn, Molecular vibration in cold-collision theory, Phys. Rev. A 65, 064702 (2002)

142. R.V. Krems, A. Dalgarno, Quantum-mechanical theory of atom-molecule and molecular collisions in a magnetic field: Spin depolarization, J. Chem. Phys. 120, 2296 (2004)

143. R.V. Krems, H.R. Sadeghpour, A. Dalgarno, D. Zgid, J. Klos, G. Chalasinski, Low-temperature collisions of $\mathrm{NH}\left(\mathrm{X}^{3} \Sigma^{-}\right)$molecules with He atoms in a magnetic field: An ab initio study, Phys. Rev. A 68, 051401(R) (2003)

144. R.V. Krems, A. Dalgarno, N. Balakrishnan, G.C. Groenenboom, Spin-flipping transitions in ${ }^{2} \Sigma$ molecules induced by collisions with structureless atoms, Phys. Rev. A 67, 060703 (2003)

145. A.V. Avdeenkov, D.C.E. Bortolotti, J.L. Bohn, Fieldlinked states of ultracold polar molecules, Phys. Rev. A 69, $012710(2004)$

146. P. Soldán, J.H. Hutson, Interaction of $N H\left(X^{3} \Sigma\right)$ Molecules with Rubidium Atoms: Implications for Sympathetic Cooling and the Formation of Extremely Polar Molecules, Phys. Rev. Lett. 92, 163202 (2004)

147. R.V. Krems, A. Dalgarno, Threshold laws for collisional reorientation of electronic angular momentum, Phys. Rev. A 67, 050704 (2003)

148. V. Aquilanti, G. Grossi, Angular momentum coupling schemes in the quantum mechanical treatment of P-state atom collisions, J. Chem. Phys. 73, 1165 (1983)

149. R.V. Krems, G.C. Groenenboom, A. Dalgarno, Electronic interaction anisotropy between atoms in arbitrary angular momentum states, J. Phys. Chem. A 108, 8941 (2004)

150. R.V. Krems, A. Dalgarno, Disalignment transitions in cold collisions of ${ }^{3} P$ atoms with structureless targets in a magnetic field, Phys. Rev. A 68, 013406 (2003)

151. V. Kokoouline, R. Santra, C.H. Greene, Multichannel cold collisions between metastable Sr atoms, Phys. Rev. Lett. 90, 253201 (2003)

152. R.V. Krems, D. Zgid, G. Chalasinski, J. Klos, A. Dalgarno, On the Possibility of Buffer Gas Cooling of Paramagnetic Carbon to Ultracold Temperatures, Phys. Rev. A 66, 030702 (2002)

153. R.V. Krems, Breaking van der Waals molecules with magnetic fields, Phys. Rev. Lett. 93, 013201 (2004)

154. E.A. Hinds, P.G.H. Sandars, Experiment to search for $P$ and T-violating interactions in the hyperfine structure of thallium fluoride, Phys. Rev. A 21, 480 (1980)

155. E.A. Hinds, K. Sangster, Testing time-reversal symmetry with molecules, AIP Conf. Proc. 270, 77 (1993)

156. E.D. Commins, Adv. At. Mol. Opt. Phys. 40, 1 (1999)

157. D. DeMille, F. Bay, S. Bickman, D. Kawall, D. Krause Jr, S.E. Maxwell, L.R. Hunter, Investigation of $\mathrm{PbO}$ as a system for measuring the electric dipole moment of the electron, Phys. Rev. A 61, 052507 (2000)

158. B.C. Regan, E.D. Commins, C.J. Schmidt, D. DeMille, New limit on the electron electric dipole moment, Phys. Rev. Lett. 88, 071805 (2002)

159. J.J. Hudson, B.E. Sauer, M.R. Tarbutt, E.A. Hinds, Measurement of the electron electric dipole moment using YbF molecules, Phys. Rev. Lett. 89, 023003 (2002)

160. N. Fortson, P. Sandars, S. Barr, The search for a permanent electric dipole moment, Phys. Today 56, 33 (2003)

161. Ch. Daussy, T. Marrel, A. Amy-Klein, C.T. Nguyen, Ch.J. Borde, Ch. Chardonnet, Limit on the parity nonconserving energy difference between the enantiomers of a chiral molecule by laser spectroscopy, Phys. Rev. Lett. 83, 1554 (1999) 